\title{
Numerical Study of the Dynamic, Thermodynamic and Microstructural Parameters of Convective Clouds
}

\author{
Vitaly A. Shapovalov ${ }^{1}$, Alexander V. Shapovalov ${ }^{1 *}$, Boris P. Koloskov², \\ Ruslan Kh. Kalov ${ }^{1}$, Valery N. Stasenko ${ }^{3}$ \\ ${ }^{1}$ Department of Cloud Physics, High-Mountain Geophysical Institute, Nalchik, Russia; ${ }^{2}$ Autonomous Nonprofit \\ Organization "Agency of Atmospheric Technologies", Moscow, Russia; ${ }^{3}$ State research center on Space \\ Hydrometeorology PLANETA, Moscow, Russia
}

Correspondence to: Alexander V. Shapovalov, atajuk@mail.ru

Keywords: Numerical 3D Modeling, Convective Processes in the Atmosphere, Thermodynamic Parameters, Microstructural Parameters, Velocity Field of Air Flow, Particles Trajectories

Received: January 16, $2018 \quad$ Accepted: February 11, $2018 \quad$ Published: February 14, 2018

Copyright $\odot 2018$ by authors and Scientific Research Publishing Inc.

This work is licensed under the Creative Commons Attribution International License (CC BY 4.0).

http://creativecommons.org/licenses/by/4.0/

\section{(c) (i) Open Access}

\section{ABSTRACT}

On the basis of a three-dimensional non stationary model of a convective cloud with detailed description of dynamic, thermodynamic and microphysical processes, numerical experiments were conducted to study the formation of parameters of convective clouds under unstable stratification of the atmosphere. Numerical experiments have been carried out to study the formation of convective processes in the atmosphere. The thermo hydrodynamic parameters in the zone of a thunderstorm cloud are determined, and regions with a vortex motion of air are identified. The main flows feeding the convective cloud in the mature stage are determined. Due to the means of visualization, the areas of formation and growth of precipitation particles are identified. In a three-dimensional form, the interaction of dynamic and thermodynamic processes is analyzed. The interaction of fields is manifested in the form of deformation of fields of thermodynamic parameters under the influence of dynamic processes. Trajectories of air streams around a cloud and the trajectories of drops in a cloud are determined. The results of numerical experiments confirm that dynamic processes significantly influence the formation of fields of thermodynamic parameters in the cloud, which also determine the course of microphysical processes and the nature of the growth of precipitation particles.

\section{INTRODUCTION}

The development of physics of convective clouds and cloud seeding at the present stage requires the solution of a number of large and interrelated tasks of fundamental and applied character, which are 
complex research. Cloud formation and development processes are main of them, as include cloud clusters formation and interaction of clouds with surrounding atmosphere [1-3]. This is due to the fact that a convective cloud is a highly complex thermodynamic and microphysics system, important features of which are the nonstationarity, three-dimensionality and nonlinearity [3-7]. Therefore, the solution of problems of physics of convective clouds requires a holistic approach and the use of more effective methods, the most important among which is numerical modeling [1-10].

\section{MATERIALS AND METHODS}

The aim of this work is the study on the basis of three-dimensional numerical model of convective clouds the formation of dynamic, thermodynamic and microstructural characteristics, and interaction of physical processes in clouds.

The subject of study is a three-dimensional convective cloud parameters: potential temperature, specific humidity, air flow, turbulence and distribution of drops and ice particles.

In the work briefly described is a three-dimensional non stationary model of convective cloud with detailed account for the thermodynamic, microphysical and electric processes.

The model includes the equations of hydrothermodynamics, microphysics and electrical processes that were used in the works [1-3].

Thermodynamic block of model consists of the equations of motion that describe moist convection in the Boussinesq approximation, which takes into account advective and turbulent transport, buoyancy force, friction and pressure gradients:

$$
\begin{gathered}
\frac{\partial u}{\partial t}+(\boldsymbol{V} \cdot \nabla) u=-\frac{\partial \pi^{\prime}}{\partial x}+\Delta^{\prime} u+l v, \\
\frac{\partial v}{\partial t}+(\boldsymbol{V} \cdot \nabla) v=-\frac{\partial \pi^{\prime}}{\partial y}+\Delta^{\prime} v-l u, \\
\frac{\partial w}{\partial t}+(\boldsymbol{V} \cdot \nabla) w=-\frac{\partial \pi^{\prime}}{\partial z}+\Delta^{\prime} w+g\left(\theta^{\prime} / \theta_{0}+0.61 s^{\prime}-Q_{S}\right),
\end{gathered}
$$

the equation of continuity

$$
\frac{\partial u}{\partial x}+\frac{\partial v}{\partial y}+\frac{\partial w}{\partial z}=\sigma w
$$

the equations of thermodynamics

$$
\begin{gathered}
\frac{\partial \theta}{\partial t}+(\boldsymbol{V} \cdot \nabla) \theta=\frac{L_{k}}{C_{p}} \frac{\theta}{T} \frac{\delta M_{k}}{\delta t}+\frac{L_{C}}{C_{p}} \frac{\theta}{T} \frac{\delta M_{C}}{\delta t}+\frac{L_{3}}{C_{p}} \frac{\theta}{T} \frac{\delta M_{3}}{\delta t}+\Delta^{\prime} \theta, \\
\frac{\partial s}{\partial t}+(\boldsymbol{V} \cdot \nabla) s=-\frac{\delta M_{k}}{\delta t}-\frac{\delta M_{C}}{\delta t}+\Delta^{\prime} s,
\end{gathered}
$$

where $(\boldsymbol{V} \cdot \nabla) \equiv u \frac{\partial}{\partial x}+v \frac{\partial}{\partial y}+w \frac{\partial}{\partial z}, \Delta^{\prime}=\frac{\partial}{\partial x} K \frac{\partial}{\partial x}+\frac{\partial}{\partial y} K \frac{\partial}{\partial y}+\frac{\partial}{\partial z} K \frac{\partial}{\partial z}$,

$\boldsymbol{V}=\{u, v, w\}$-velocity vector, $u(\boldsymbol{r}, t), v(\boldsymbol{r}, t), w(\boldsymbol{r}, t)$-components of the velocity vector of air flow in the cloud; $\theta(\boldsymbol{r}, t)$-potential temperature; $\pi(\boldsymbol{r}, t)=c_{p} \bar{\theta}(P(\boldsymbol{r}, t) / 1000)^{R / C_{p}}$ the dimensionless pressure; $\bar{\theta}$ the average potential temperature; $R$-gas constant; $s(\boldsymbol{r}, t)$ the specific humidity of air, $Q_{S}(\boldsymbol{r}, t)$ the total mixing ratio of liquid and solid phases in the cloud; $\sigma(z)$-a parameter that takes into account the change of air density with height; $P(\boldsymbol{r}, t)$ and $T(\boldsymbol{r}, t)$ the pressure and the temperature, respectively; $C_{p}$ the heat capacity of air at constant pressure; $L_{C}, L_{S}, L_{F}$ the specific heat of condensation, sublimation and freezing, respectively; $\pi^{\prime}(\boldsymbol{r}, t), \theta^{\prime}(\boldsymbol{r}, t), s^{\prime}(\boldsymbol{r}, t)$-deflection dimensionless pressure, potential temperature and specific humidity from their background values in ambient atmosphere 
$\pi_{0}(\boldsymbol{r}), \theta_{0}(\boldsymbol{r}), \mathrm{s}_{0}(\boldsymbol{r}) ; \frac{\delta M_{C}}{\delta t}, \frac{\delta M_{S}}{\delta t}$ the changes in specific humidity due to diffusion of vapor on the drops and crystals; $\frac{\delta M_{F}}{\delta t}$ the mass of frozen water droplets in unit time in unit volume of air; $K(\boldsymbol{r}, t)$ the turbulent diffusion coefficient; $l$ the Coriolis parameter. The vector $\boldsymbol{r}$ corresponds to the coordinates $(x, y, z)$. For the boundaries of the spatial domain symbols $0, L_{x}, 0, L_{y} 0, L_{z}$ are used.

Equations for the distribution functions of droplets and crystals by mass:

$$
\begin{aligned}
& \frac{\partial f_{1}}{\partial t}+u \frac{\partial f_{1}}{\partial x}+v \frac{\partial f_{1}}{\partial y}+\left(w-V_{1}\right) \frac{\partial f_{1}}{\partial z} \\
& =\left(\frac{\partial f_{1}}{\partial t}\right)_{C}+\left(\frac{\partial f_{1}}{\partial t}\right)_{C G}+\left(\frac{\partial f_{1}}{\partial t}\right)_{A C}+\left(\frac{\partial f_{1}}{\partial t}\right)_{D R}+\left(\frac{\partial f_{1}}{\partial t}\right)_{F R}+\Delta^{\prime} f_{1}+I_{1} \\
& \frac{\partial f_{2}}{\partial t}+u \frac{\partial f_{2}}{\partial x}+v \frac{\partial f_{2}}{\partial y}+\left(w-V_{2}\right) \frac{\partial f_{2}}{\partial z} \\
& =\left(\frac{\partial f_{2}}{\partial t}\right)_{S B}+\left(\frac{\partial f_{2}}{\partial t}\right)_{A C}+\left(\frac{\partial f_{2}}{\partial t}\right)_{F R}+\Delta^{\prime} f_{2}+I_{2}
\end{aligned}
$$

$\left(\frac{\partial f_{1}}{\partial t}\right)_{C},\left(\frac{\partial f_{1}}{\partial t}\right)_{C G},\left(\frac{\partial f_{1}}{\partial t}\right)_{A C},\left(\frac{\partial f_{1}}{\partial t}\right)_{D R},\left(\frac{\partial f_{1}}{\partial t}\right)_{F R}$-changes of the distribution functions of droplets due to microphysical condensation processes, coagulation of droplets, accretion of droplets and crystals, crushing and freezing of drops, respectively; $\left(\frac{\partial f_{2}}{\partial t}\right)_{S B},\left(\frac{\partial f_{2}}{\partial t}\right)_{A C},\left(\frac{\partial f_{2}}{\partial t}\right)_{F R}$-changes of the distribution functions of crystals due to sublimation, accretion and freezing of droplets; $I_{1}$ and $I_{2}$-sources of droplets and crystals.

Initial and boundary conditions for Equations (1)-(4) are as follows:

$$
\begin{gathered}
u(\boldsymbol{r}, 0)=u_{0}(\boldsymbol{r}), v(\boldsymbol{r}, 0)=v_{0}(\boldsymbol{r}), w(\boldsymbol{r}, 0)=w_{0}(\boldsymbol{r}), \\
\theta(\boldsymbol{r}, 0)=\theta_{0}(\boldsymbol{r}), s(\boldsymbol{r}, 0)=s_{0}(\boldsymbol{r}), \\
f_{1}(\boldsymbol{r}, m, 0)=f_{2}(\boldsymbol{r}, m, 0)=f_{3}(\boldsymbol{r}, m, 0)=0, \\
u(\boldsymbol{r}, t)=u_{0}(\boldsymbol{r}), v(\boldsymbol{r}, t)=v_{0}(\boldsymbol{r}), w(\boldsymbol{r}, t)=w_{0}(\boldsymbol{r}), \\
\theta(\boldsymbol{r}, t)=\theta_{0}(\boldsymbol{r}), s(\boldsymbol{r}, t)=\left.s_{0}(\boldsymbol{r})\right|_{x=0, L_{x} ; y=0, L_{y} ; z=L_{z}} \\
u(\boldsymbol{r}, t)=v(\boldsymbol{r}, t)=w(\boldsymbol{r}, t)=0, \theta(\boldsymbol{r}, t)=\theta_{0}(\boldsymbol{r}), s(\boldsymbol{r}, t)=\left.s_{0}(\boldsymbol{r})\right|_{z=0} \\
\quad f_{1}(\boldsymbol{r}, m, t)=f_{2}(\boldsymbol{r}, m, t)=\left.0\right|_{x=0, L_{x} ; y=0, L_{y} ; z=L_{z}} \\
\quad \partial f_{1}(\boldsymbol{r}, m, t) / \partial z=\partial f_{2}(\boldsymbol{r}, m, t) / \partial z=\left.0\right|_{z=0}
\end{gathered}
$$

Microphysical block of the model describes the processes of nucleation, condensation, coagulation of the droplets with the droplets, sublimation, accretion, freezing of drops, sedimentation of cloud particles in a gravity field, the airflow transfer [2].

The system of equations of model was solved by the method of splitting into physical processes and component splitting. In the first stages of the procedure of splitting the equations of hydrothermodynamics are solved. One common approach to the numerical solution of the dynamics equations 
is based on the solution of an elliptic equation for the pressure. Firstly the system of equations of the wind and turbulent change of fields of airflows was solved. Derived fields are not adapted. To obtain the final values the Poisson equation for the pressure field is solve, we used an iterative method [3].

Let consider some of the results of studies of dynamic and microstructural parameters of convective clouds under different stratifications of the atmosphere.

The size of the spatial region in the calculations was set $40 \times 40 \mathrm{~km}$ horizontal and $16 \mathrm{~km}$ in the vertical. The grid spacing in the $\mathrm{X}, \mathrm{Y}$ coordinates was $500 \mathrm{~m}$, along the $\mathrm{Z}-250 \mathrm{~m}$. The $\mathrm{X}$ axis is directed eastward, $\mathrm{Y}$-northward. The cloud was initiated by the set pulse at the earth surface with the overheating $\Delta \mathrm{T}=1.0^{\circ} \mathrm{C}$. The shape and size of the pulse was varied.

For the analysis of calculation results the software for three-dimensional data visualization was developed, which allows one to see the modeling object, rotate it in the vertical and horizontal plane, to change the form of representation: isosurfaces, isolines, vector field, etc. One can also quickly change the displayed value of the parameter by moving the slider in the program menu.

The first phase of research was aimed at modeling of the development of axisymmetric clouds in a calm atmosphere to analyze the quality of the model and algorithms of calculations. The data showed high accuracy of numerical algorithms developed for the model.

Hereinafter let we consider the results of calculations of dynamic and microstructure parameters of clouds in different stages.

\section{RESULT AND DISCUSSION}

The simulation results show that the dynamic, thermodynamic and microphysical parameters in convective clouds exert a mutual influence on each other. Figure 1 shows the airflows in a vertical plane passing through the cloud. Radar reflectivity volume (a value of $10 \mathrm{dBZ}$ ) is made translucent for flows analysis inside the cloud. It is noted the presence of the vortex on the windward side of the cloud (on the left in Figure 1) and with downwind.

During convective clouds simulations, downward streams of air around the clouds were observed. On the windward side, they can be amplified by an external wind, and on the leeward side they may be weaker due to the hydrodynamic shade in this part of the cloud.

Figure 2 shows the results of calculations of airflows in the cloud and around the cloud on which the noticeable downward flows are observed around the cloud.

Numerical experiments based on the upper air balloon sounding showed that the characteristics of the result clouds are sensitive to the vertical profiles of temperature and humidity. Quite powerful clouds were observed upon significant instability of the atmosphere, the tops of which consisted of crystals. When model clouds were compared to actual ones, qualitative and quantitative agreement was noticed.

The lines of airflows that run into the cloud in the maturity stage (Figure 3 ) were identified using the

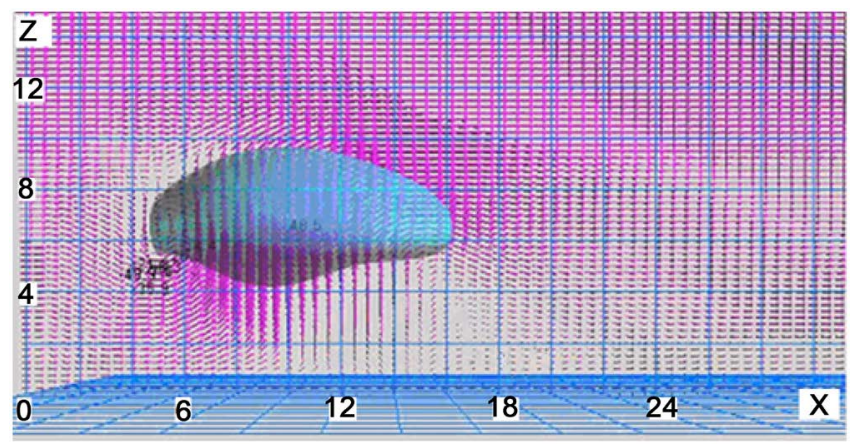

Figure 1. Velocity field of air flow in the vertical plane (XZ) passing through the cloud. Values are represented as vectors. The isosurface (volume) of weather radar reflectivity (with a value of $10 \mathrm{dBZ}$ ) is visible as translucent. The horizontal and vertical grids with cells of $2 \times 2 \mathrm{~km}$ are shown. 


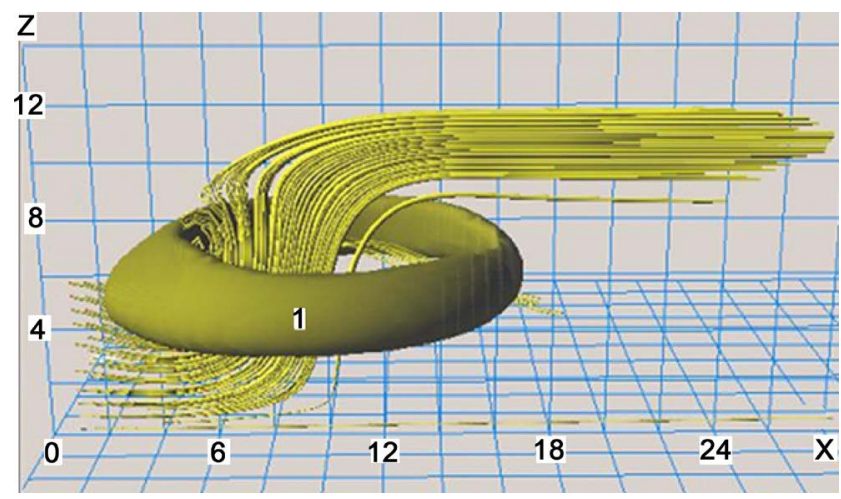

Figure 2. Upward (bundle of streamlines) and downward (isosurface 1) airflows in the convective cloud on the $35^{\text {th }}$ minute of the development. The horizontal and vertical grids with cells of $2 \times 2 \mathrm{~km}$ are shown.

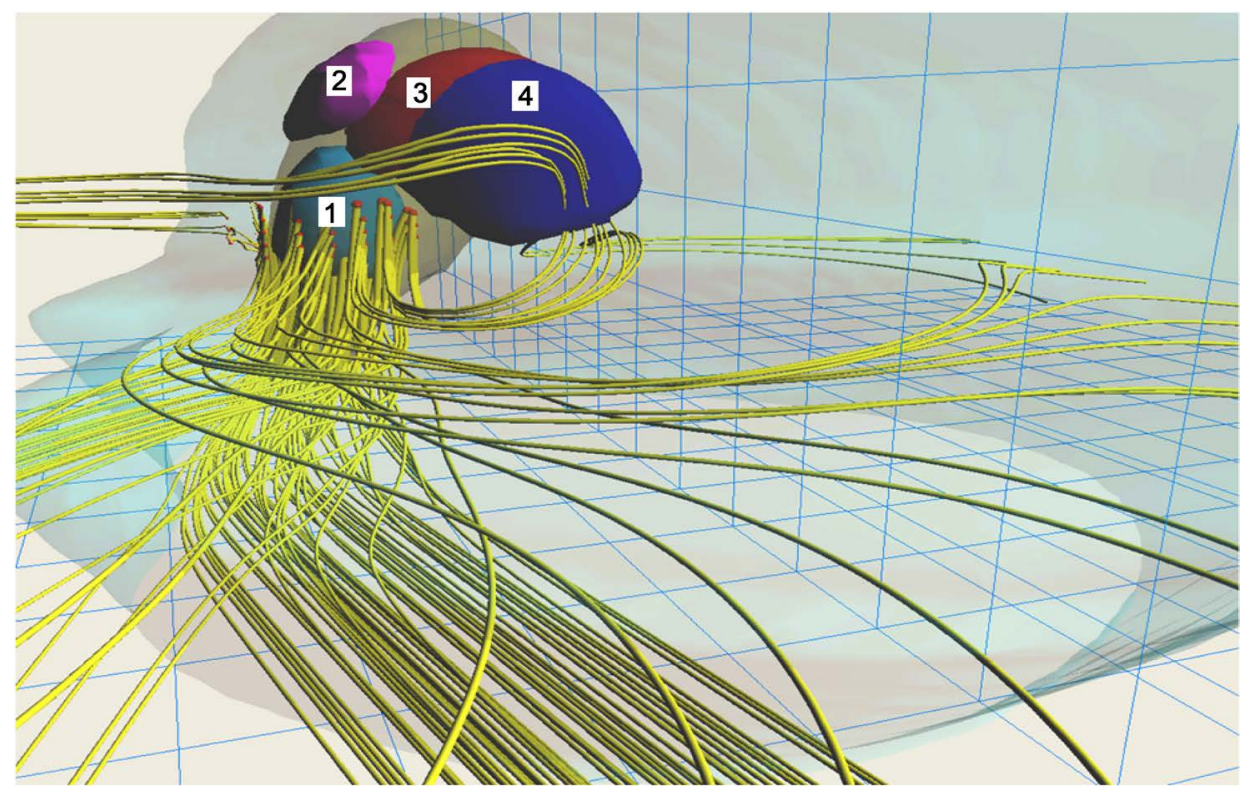

Figure 3. Air flows (streamlines) at the mature stage of the convective cloud. On the picture there are: the zone of formation of the large ice particles and here also recirculation zone-4; large and medium size water drops-1; small size ice particles-2; medium size ice particles-3, respectively.

developed visualization tools. The figure shows several preferential flows from several directions: from the right side in the direction of cloud movement, from the rear part of the cloud, a small beam in the direction of the average wind in the atmosphere.

The numerical simulation of cloud let us to make space and time analysis of vertical and horizontal velocity of airflows, the turbulent diffusion coefficient, water content, ice content and other parameters on different stages of the cloud. Figure 4 shows some characteristics of the simulated convective cloud with isosurfaces for the large drops $(\mathrm{r}>100 \mu \mathrm{m})$ and ice particles $(\mathrm{r}>100 \mu \mathrm{m})$, trajectory of the some droplets in the cloud (3), accompanied by the several contours of radar reflectivity.

\section{CONCLUSION}

Three-dimensional non-stationary numerical model of a convective cloud with detailed description of thermodynamic and microphysical processes was developed. 


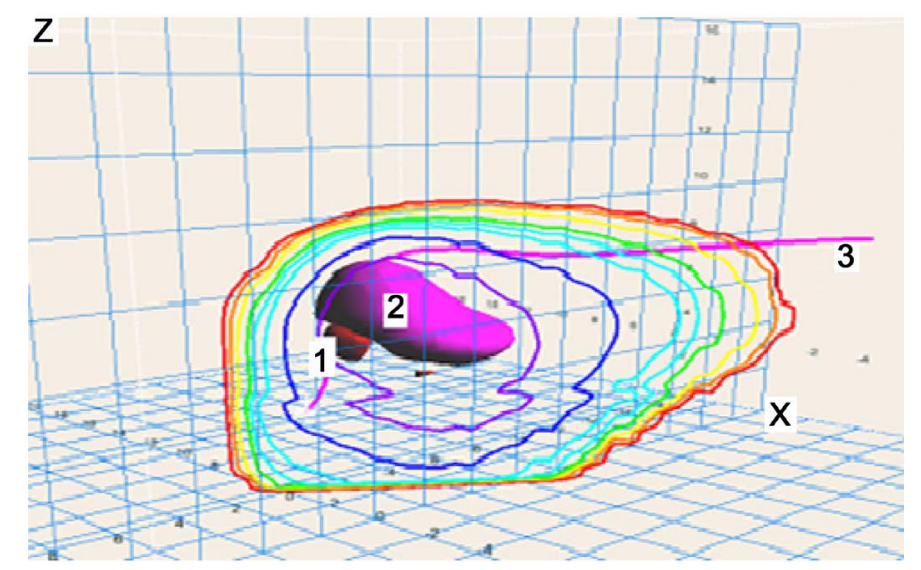

Figure 4. Some characteristics of the simulated convective cloud (of the $10 \mathrm{~km}$ height on the 30th minute of evolution) with volumes of large drops (1), large ice particles (2), several contours of radar reflectivity (with an interval of $5 \mathrm{dBZ}$ ) and one of the possible trajectories of drops in the cloud (3).

The special software is developed for 3-dimensional visualization of simulation data. The software includes convenient tools for detailed analysis of the thermodynamic parameters and microstructure of the cloud.

Numerical experiments on research of formation of convective processes in the atmosphere under different stratifications of the atmosphere and the background wind were conducted. Thermodynamic parameters in a zone of thunderstorm clouds were determined in the areas with the vortex motion of the air. The main streams that feed the deep cloud in the mature stage were defined.

By means of visualization tools, regions of the formation and growth of precipitation particles were identified.

The interaction of dynamic and thermodynamic processes was analyzed in 3D. The interaction of the different physical processes is manifested in the form deformation of fields of thermodynamic parameters under the influence of dynamic processes. Cloud simulation furthers to track air flows around the cloud and the trajectories of the droplets in the cloud.

The results of numerical experiments confirm that dynamic processes significantly affect the formation of fields of thermodynamic parameters in the cloud that in turn determine the microphysical processes and the nature of the growth of precipitation particles.

\section{REFERENCES}

1. Ashabokov, B.A. and Shapovalov, A.V. (2008) Convective Clouds: Numerical Models and Simulation Results in Natural Conditions and the Active Impact. Kabardino-Balkaria Science Center of RAS Press, Nalchik, $254 \mathrm{p}$.

2. Kogan, E.A., Mazin, I.P., Sergeev, B.N. and Khvorostyanov, V.I. (1984) Numerical Simulation of Clouds. Gidrometeoizdat Press, Moscow, 185 p.

3. Kupovykh, G.V., Ashabokov, B.A., et al. (2012) Numerical Simulation of the Electrical Characteristics of Convective Clouds. Proceedings of the Universities. The North Caucasus Region. Natural Science, 6, 65-68.

4. Ashabokov, B.A., Shapovalov, A.V., Kuliev, D.D., Prodan, K.A. and Shapovalov, V.A. (2014) Numerical Simulation of Thermodynamic, Microstructural, and Electric Characteristics of Convective Clouds at the Growth and Mature Stages. Radiophysics and Quantum Electronics, 56, 811-817. https://doi.org/10.1007/s11141-014-9483-z

5. Fan, J., et al. (2009) Dominant Role by Vertical Wind Shear in Regulating Aerosol Effects on Deep Convective Clouds. Journal of Geophysical Research: Atmospheres, 114, Issue D22. https://doi.org/10.1029/2009JD012352

6. Karacostas, T., Spiridonov, V., Bampzelis, D., Pytharoulis, I., Tegoulias, I. and Tymbanidis, K. (2016) Analysis 
and Numerical Simulation of a Real Cell Merger Using a Three-Dimensional Cloud Resolving Model. Atmospheric Research, 169, 547-555. https://doi.org/10.1016/j.atmosres.2015.09.011

7. Khain, A., Prabha, T.V., Benmoshe, N., Pandithurai, G. and Ovchinnikov, M. (2013) The Mechanism of First Raindrops Formation in Deep Convective Clouds. Journal of Geophysical Research: Atmospheres, 118, 9123-9140. https://doi.org/10.1002/jgrd.50641

8. Khvorostyanov, V.I. and Curry, J.F. (2014) Thermodynamics, Kinetics, and Microphysics of Clouds. Cambridge University Press, New York, 777 p. https://doi.org/10.1017/CBO9781139060004

9. Iguchi, T., Matsui, T., Shi, J.J., Tao, W.-K., Khain, A.P., Hou, A., Cifelli, R., Heymsfield, A. and Tokay, A. (2012) Numerical Analysis Using WRF-SBM for the Cloud Microphysical Structures in the C3VP Field Campaign: Impacts of Supercooled Droplets and Resultant Riming on Snow Microphysics. Journal of Geophysical Research: Atmospheres, 117, Issue D23. https://doi.org/10.1029/2012JD018101

10. Liu, X.L. and Niu, S.J. (2010) Numerical Simulation of Macro- and Micro-Structures of Intense Convective Clouds with a Spectral Bin Microphysics Model. Advances in Atmospheric Sciences, 27, 1078-1088.

https://doi.org/10.1007/s00376-010-8088-5 